\title{
Virtual Earthquake Engineering Laboratory Capturing Nonlinear Shear, Localized Damage and Progressive Buckling of Bar
}

\author{
In Ho Cho ${ }^{\text {a) }}$ M.EERI
}

\begin{abstract}
We embarked upon developing a novel parallel simulation platform that is rooted in microphysical mechanisms. Primarily aiming at large-scale reinforced-concrete structures exposed to cyclic loading, we sought to settle the question as to how to capture nonlinear shear, localized damage and progressive buckling of reinforcing bar. We proposed a tribology-inspired three-dimensional (3-D) interlocking mechanism in the well-established framework of multidirectional smeared crack models. Strong correlation between random material property and localized damage has been shown, notably at the global system level. An automated platform has been suggested to capture progressive buckling phenomena. Validation and applications straddle a wide range, from small laboratory tests to large-scale 3-D experiments, successfully offering a clear causal pathway between underlying physical mechanisms and the unresolved issues addressed above. [DOI: 10.1193/1.4000095]
\end{abstract}

\section{INTRODUCTION}

In the earthquake engineering community, numerical simulation has played an essential role in increasing resilience of infrastructures, providing the optimal rehabilitation remedy, and even generating precise probabilistic fragility curves in the loss estimation process. Still, we have seen a substantial gap between our simulation capability and actual damage and failure modes of real structures during recent big earthquakes. Filling the gap by harmoniously combining physics-based mechanisms and cutting-edge parallel computers was the original motivation of our novel attempt.

The challenges that we are trying to tackle herein include the nonlinear shear, localized damage and progressive buckling of reinforcing bar. Indeed, those issues have long been completely unresolved, and are closely tied to the limitations of state-of-the-art simulation technology of earthquake engineering fields.

For describing nonlinear behavior of shear-critical reinforced-concrete (RC) structural members, a large number of numerical and theoretical models have been proposed, which proved successful in providing important insight into global nonlinear responses. Some works are based on well-designed panel tests (Mansour and Hsu 2005, Hsu and Mansour 2005, Vecchio 1999), while the popular fiber section models are often employed by others (Orakcal and Wallace 2006, Yazgan and Dazio 2011). Even a simple multilinear

a) Depart. of Mechanical and Civil Engineering, California Institute of Technology, Pasadena, CA 91125 
moment-curvature relationship has been effectively utilized for particular cases where flexural behavior is dominant (Adebar and Ibrahim 2002).

Although all of these attempts proved powerful in understanding salient nonlinear behavior of shear-critical RC structural members, most of them fundamentally rely on particular types of experimental conditions such as well-controlled force and displacement boundary conditions or geometric simplicity. Also, the limitation of the popular fiber section approach is well recognized - the key restrictive assumption that plane section remains plane after deformation. More importantly, localized damages, which exert strong influence on nonlinear degradation of RC systems, are often dealt with by some averaged quantities, normally reflected from global force-displacement response. But, in reality, the localized failure modes appear to emerge in various ways depending on mechanical characteristics of RC members under earthquake loadings (e.g., Hanson 1996).

The other challenge can be found in steel bar models. To date, a multitude of attempts have been made to describe nonlinear behavior of reinforcing bars under cyclic loading. For instance, Kunnath et al. (2009) proposed a generic model by incorporating the initiation of compressive buckling, low-cycle fatigue fracture, and cyclic strength degradation. Focused on postyield buckling, Dhakal and Maekawa (2002a) suggested a simple model requiring only a few parameters, and they derived an analytical method for estimating buckling length (Dhakal and Maekawa 2002b). Rodriguez et al. (1999) paid considerable attention to the postbuckling stress-strain behavior of reinforcing bars. Monti and Nuti (1992) combined plasticity theory and an empirical buckling model so as to cover various hardening models. Pantazopoulou (1998) pointed out the significant role of the properties of the surrounding system. Bae et al. (2005) presented intriguing factors such as initial geometric imperfection and the ratio of the ultimate strength to the yield strength.

Despite such valuable accomplishments, the aforementioned formulations regarding compressive buckling tend to rely on common assumptions - such as idealized deformation shape of a short bar, firmly fixed ties, and two ends of bar allowing no horizontal displacement. In reality, however, the initial alignment of a reinforcing system deforms in a very complicated way due to the rigorous interaction with the surrounding system of cover and core concrete, horizontal steels such as ties and stirrups, and other longitudinal steels. Hence the progressive buckling phenomenon essentially calls for a comprehensive and holistic approach to crushing/spalling of brittle material, yielding and lengthened buckling length of reinforcing bars, and so on.

One might suggest that we can simply perform highly detailed three-dimensional analysis because powerful parallel computing resources (e.g., OpenSeesSP and OpenSeesMP) have become available in our field. Indeed, with such advanced parallel computing technologies at hand, popular continuum-based plasticity models - for example, quasi-brittle model by Simo and Ju (1987a, b), ductile material under cyclic loading by Steglich et al. (2005), localized shear band model by Ortiz (1987) and Jirásek (1998) - could be successful candidates to resolve the challenges of nonlinear shear and localized damage.

However, it should be stressed that those continuum-based models are often handicapped by the sensitivity to tangent stiffness (thus causing convergence problems), the lack of 
physical counterparts of salient parameters, and the difficulty in handling evolution of yielding surface under cyclic loading.

Thus we embarked upon developing a novel parallel simulation platform. Our first set of goals include: (1) delineating nonlinear shear behavior by means of a reasonable microphysical mechanism, (2) capturing progressive buckling of reinforcing bar, and (3) detecting localized damage at the global system level.

Toward this end, we shall first revisit the "fixed-type" multidirectional smeared crack model, which had been adopted to fully retain the physical nature of cracks. Next we will discuss the tribology-inspired 3-D interlocking model, which is responsible for generating accurate nonlinear shear resistance and resolving pathological nature of the "fixed-type" crack model. Then, we shall touch upon a novel automated platform for modeling progressive buckling of reinforcing bars by comprehensive consideration of the reinforced system as a whole.

Validations and applications straddle a wide range from small laboratory tests to largescale RC structures exposed to severe cyclic loading. Notably, clear detection of localized damage shall be presented, which appears to be related to the randomness over the entire domain. It is noteworthy that behind all of the novelty lies the cutting-edge parallel computing technology (see Cho and Hall 2012).

\section{REVISITING THE "FIXED-TYPE" MULTIDIRECTIONAL SMEARED CRACK MODEL}

The fixed-type smeared crack model is regarded to best retain the physical attribute of crack, since the primary crack surface remains unchanged by subsequent loadings. The downside of the fixed crack model, however, is also well known - spurious large stress development under nonproportional loading. This deleterious attribute, therefore, brought many supplementary alternatives after Rashid (1968) - for example, de Borst and Nauta (1985), Weihe et al. (1998), and even rotating crack models (Cope et al. 1980, Rots 1988 and Jirásek and Zimmermann 1998). Since we placed top priority on preserving physical counterparts, we adopted the fixed-type smeared crack model rather than the rotating crack model.

The present smeared crack model allows three orthogonal crack surfaces and utilizes total strain. For comparable approaches, refer to such various works as Vecchio and Collins (1986), and Selby and Vecchio (1993, 1997); the clear contrast to their works lies in the scale of the present crack model, which is assumed to be far smaller than that used in those practical smeared crack models. For detailed flow of the fixed-type smeared crack model, see the references above or the author's thesis (Cho 2012).

For updating microscopic stresses on the three crack surfaces, we integrated the advantageous attributes of the previous accomplishments of tension/compression models (see Figure 1) - that is, we adopted the Thorenfeldt et al. concrete model (1987), which is augmented by the linear re/unloading model of Taucer et al. (1991) and Moelands and Reinhardt's model (Reinhardt 1984) with $c=0.31$ for nonlinear tensile softening regime. 


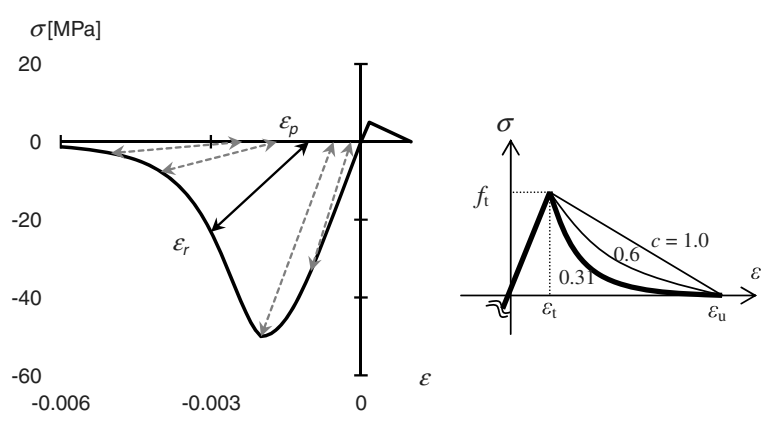

Figure 1. Microscopic stress functions $\left(\varepsilon_{r}\right.$ and $\varepsilon_{p}=$ strains defining un-/reloading path; $f_{t}$ and $\varepsilon_{t}=$ tensile strength and associated strain; $\varepsilon_{u}=$ ultimate strain with tensile resistance; $c=$ softening parameter; origin of each model is provided in the text).

It is important to note that the mesh objectivity regarding tensile crack can be realized by adopting the notion of "crack band width" $h$ (e.g. Bažant and Oh 1983) such that

$$
\int_{\varepsilon_{t}}^{\varepsilon_{u}} g \mathrm{~d} \varepsilon=G_{f}^{I} / h
$$

where $g$ is the microscopic stress function; $G_{f}^{I}$ is Mode-I fracture energy in $\left[\mathrm{J} / \mathrm{m}^{2}\right]$; and $h$ is crack band width in meters.

Similar to the trend of tribology (the science and engineering of interaction between relatively moving surfaces), the pioneering work of 2-D interlocking models by Walraven (1994) can be characterized not only by the stress transfer over opened crack directly depending on contacting areas between 2-D semicircle and its indentation, but also by a probabilistic method used for realizing rough crack surface. Inspired by tribology as well as the 2-D interlocking model, we have derived a 3-D interlocking model for describing the degradation of tangent shear resistance at the cracked material. Figure 2 shows the base couple of rigid particle-soft matrix, while the inset compares a typical asperity model consisting of rigid plate and soft hemisphere widely used in tribology (after Greenwood and Williamson 1996). The single asperity model in tribology assumes that the plastic deformation happens on the hemisphere part due to severe contact with rigid flat plate (e.g., Jackson and Green 2005). Conversely, in the present work, it is assumed that the permanent plastic deformation
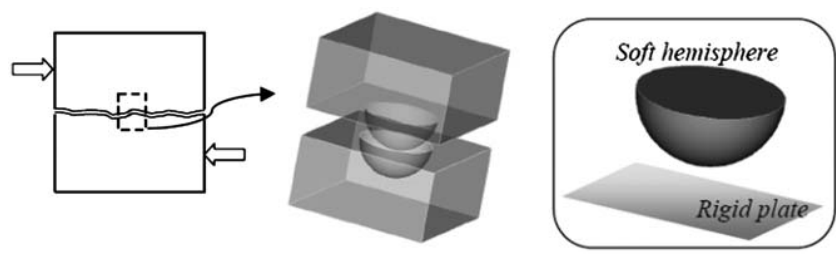

Figure 2. The base couple of rigid particle-soft matrix for 3-D interlocking model being proposed herein; (inset) typical asperity model in tribology, presented for comparison. 


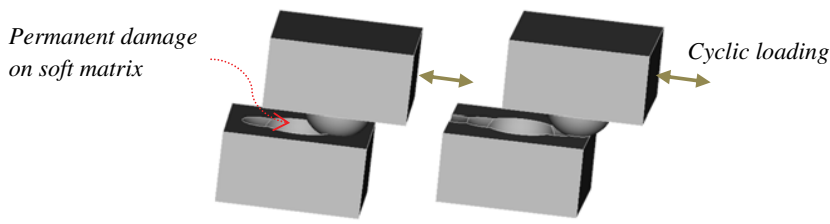

Figure 3. Permanent plastic damage on the soft matrix by cyclic movement.

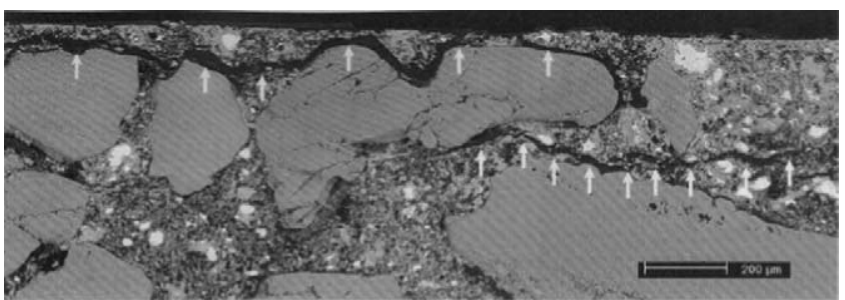

Figure 4. Micrographs revealing micro-cracking along the aggregate boundaries of a concrete specimen that is caused by severe compression (actual cross section of concrete specimen showing crack patterns emphasized by arrows; from Elkadi (2005) with permission from Delft University Press).

occurs at the soft matrix part only, and the ideal sphere remains intact during the whole cyclic loading process (see Figure 3).

The rigid particle-soft matrix assumption is strongly tied to the physical nature of microcracking. As shown in Figure 4, for instance, of concrete under excessive compression, the micro-cracking tends to develop in the weak matrix first, and to grow along the boundaries of relatively stiff aggregates. Such irregular, zigzagged configurations of opened crack surfaces can be realistically described by a large number of the fabrics of rigid sphere-soft indentation.

\section{DERIVATION OF NONLINEAR SHEAR RESISTANCE}

Using the physics-rooted mechanism addressed above, we have obtained a calcuation for nonlinear shear resistance as follows. Let us assume that one ideal particle of diameter $D_{\max }$ causes shear resistance. If it is displaced by $d$ relative gap distance between crack surfaces, we can estimate the horizontal and vertical projections of contact area of the moving sphere in terms of $d$ (see shaded area in Figure 5).

With $c=\sqrt{\left(D_{\max } / 2\right)^{2}-d^{2}}$ as depicted in Figure 5, we obtain the projected areas of hemisphere as:

$$
A_{h}=\pi c^{2} / 2 ; \quad A_{v}=\left(D_{\max } / 2\right)^{2} \arctan (c / d)-c \times d
$$

By virtue of the persistently retained direction of cracks, notably with fixed-type cracks, the tangent shear stiffness can be thought to be directly affected by contacting areas:

$$
G \propto\left(A_{v}+\mu A_{h}\right)
$$




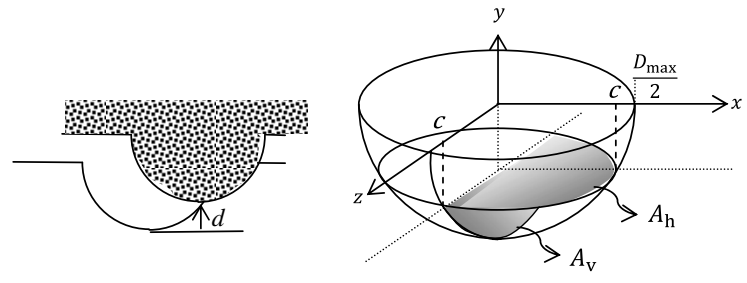

Figure 5. Projections of contact area of the ideal hemisphere with diameter $D_{\max }$.

This proportionality of shear resistance to actively contacting area is rooted in the central idea of previous research (Walraven 1994), in which total frictional force in the horizontal direction is assumed to be solely determined by the contacting area of the 2-D semicircle and its indentation.

For a concise form, after introducing $\tilde{\varepsilon}=2 d / D_{\max }$, normalization with $\left(D_{\max } / 2\right)^{-2}$ yields

$$
G \propto\left\{\arctan \sqrt{\tilde{\varepsilon}^{-2}-1}-\tilde{\varepsilon} \sqrt{1-\tilde{\varepsilon}^{2}}+\frac{\pi}{2} \mu\left(1-\tilde{\varepsilon}^{2}\right)\right\}
$$

It is natural to expect considerable difference in strength between the rigid particle and the soft matrix, which can be envisaged as the strength contrast between aggregates and cement paste for an instance of concrete. Hence appropriate reduction is to be taken into account by use of a reduction factor, denoted $C_{c s}$ herein. Although $C_{c s}$ has a physical meaning of strength difference between key constituents, determining a proper value is not tractable. Plasticity theory might be used to obtain a highly accurate value for $C_{c s}$, as is done in tribology for steel material (Jackson and Green 2005). However, considering strong heterogeneity in the materials under consideration, the empirical approach appears more attractive, and we attained $C_{c s}=1.66 \mathrm{e}-4$ from the validation against the interlocking test (Figures 910). This value was used in all numerical simulations presented here. Finally, the tangent shear stiffness on crack surface is proposed as

$$
G(\tilde{\varepsilon})=C_{c s} \frac{G_{0}}{(1+\mu)} \frac{2}{\pi}\left\{\arctan \sqrt{\tilde{\varepsilon}^{-2}-1}-\tilde{\varepsilon} \sqrt{1-\tilde{\varepsilon}^{2}}+\frac{\pi}{2} \mu\left(1-\tilde{\varepsilon}^{2}\right)\right\}
$$

where $G_{0}=$ elastic shear modulus; $\mu=$ friction coefficient $(0.4$ is used throughout the simulations); $\tilde{\varepsilon}=2 d / D_{\max }$. The choice of the fixed frictional coefficient $\mu$ has been made following the well-proven work by Walraven (1994).

We can see the relationship between $\tilde{\varepsilon}$ and $\varepsilon$ in a one-dimensional case given by

$$
\varepsilon=\frac{d}{L}=\frac{d}{D_{\max } / 2} \frac{D_{\max } / 2}{L}=\tilde{\varepsilon} \frac{D_{\max } / 2}{L}
$$

where $L=$ length of element and $\varepsilon=$ normal strain to the crack surfaces.

As seen in Figure 6, the tangent shear stiffness decreases with $\tilde{\varepsilon}$ (or equivalently with $d$ ) such that $\left.G\right|_{\tilde{\varepsilon}=0 \text { or } d=0}=C_{c s} G_{0}$ and $\left.G\right|_{\tilde{\varepsilon}=1 \text { or } d=0.5 D_{\max }}=0$. To expand on the necessity of the 


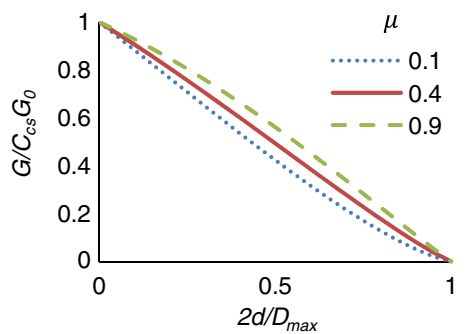

(a) 3D Hemisphere

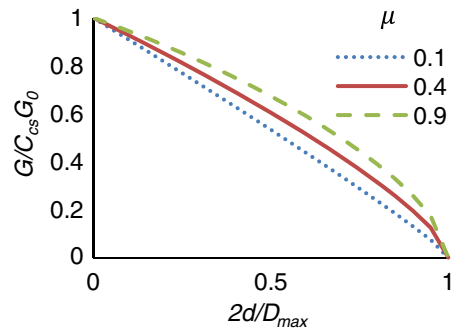

(b) 2D Semicircle

Figure 6. Degradation of tangent shear stiffness with several friction coefficients: (a) when a 3-D hemisphere is used; (b) when a 2-D semicircle is adopted for interlocking mechanism, exhibiting relatively stiff nature compared to the case with 3-D hemisphere.

3-D interlocking model in resolving the overstiffness problem of the fixed-type smeared crack model, the variation of shear stiffness obtained from the 3-D and 2-D interlocking model is compared in Figure 6. It is apparent that the 2-D semicircle-based model would reproduce relatively larger shear stiffness than the 3-D hemisphere, which would render it less favorable for alleviating the problem of overstiffness.

As shown in Figure $7 \mathrm{a}$, when the crack is completely closed, the maximum tangent shear stiffness is regained from the interlocking mechanism. It is noteworthy that once tensile crack takes place, we always employ the interlocking mechanism for evaluating nonlinear shear stiffness, which leads to the "weak" upper limit of tangent shear stiffness in the presence of Mode-I crack - cf. the "strong" upper limit proposed by Regan (1971) in which full initial shear stiffness is ideally regained after complete closure of tensile crack. In Figure 7c, we can see that if the crack fully opens more than half of the ideal particle size, there remains no resistance to shear.

In the intermediate stage when the crack begins to reopen, as depicted in Figure 7b, the shear resistance between crack surfaces is governed by the interlocking mechanism. No resistance is assumed during the unloading process, which is consistent with experimental results. During the reloading process, somewhat early resistance is gained as denoted by recontacting

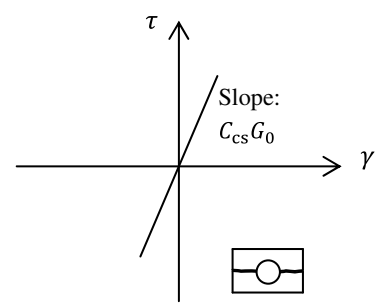

(a) $\tilde{\varepsilon} \leq 0$

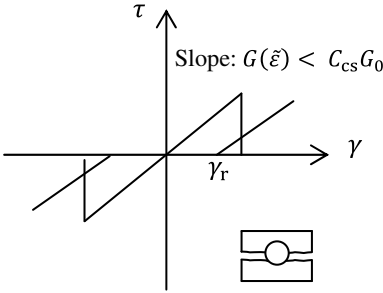

(b) $0<\tilde{\varepsilon}<1$

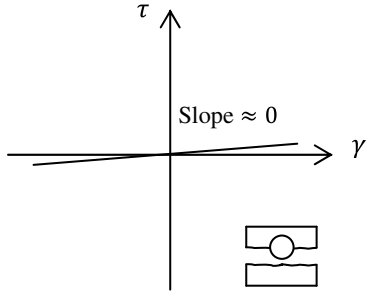

(c) $1 \leq \tilde{\varepsilon}$

Figure 7. Variation of tangent shear stiffness depending on crack opening. 


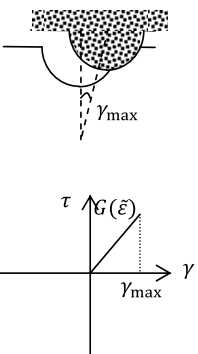

(a) Loading
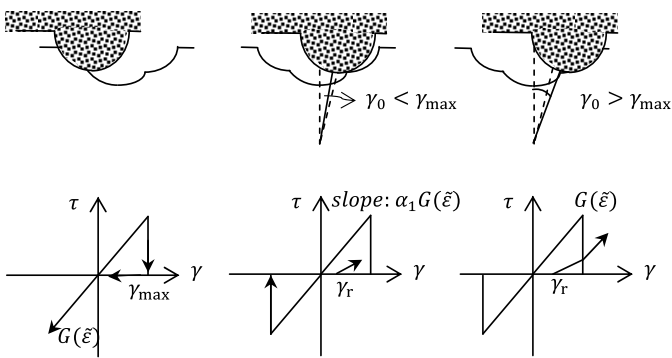

(b) Unloading

(c) Reloading

(d) Loading

Figure 8. Interlocking states during cyclic loading (all shear strain and shear stress terms signify those on the crack surfaces).

shear strain $\gamma_{r}$ in Figure $7 \mathrm{~b}$, which appears to be tied to the remaining debris of matrix left by shearing off and grinding.

However, this early resistance during reloading gradually disappears as the horizontal displacement increases, and thus an expression for $\gamma_{r}$ has been suggested such that $\gamma_{r} \rightarrow \gamma_{\max }$ as $\gamma>>0$

$$
\gamma_{r}=\left|\gamma_{\max }\right| \times\left\{a+b\left(1-\exp \left(-c \times\left|\gamma_{\max }\right|\right)\right)\right\} \quad \text { for } \quad \gamma>0
$$

where $a, b$, and $c$ are positive constant with $a+b=1$ and $\gamma_{\max }\left(\gamma_{\min }\right)$ is the maximum (minimum) shear strain ever experienced on the crack surfaces. Some recommendable values (i.e., $a=0.8 ; b=0.2 ; c=1.0$, which have been empirically calibrated from the interlocking experimented presented herein (Figures 9-10)) have shown favorable performance throughout all simulations presented herein. It is of practical importance to note that $\gamma_{\max }\left(\gamma_{\min }\right)$ is not a fixed value but rather a constantly updated value as time proceeds during nonlinear analysis. Also, the exponential decay of $\gamma_{r}$ is rooted in the knowledge from the well-documented

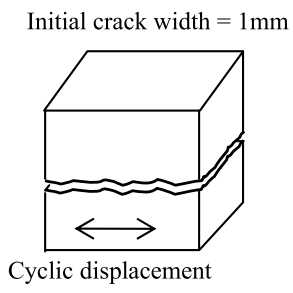

(a) Specimen with Mode-I crack

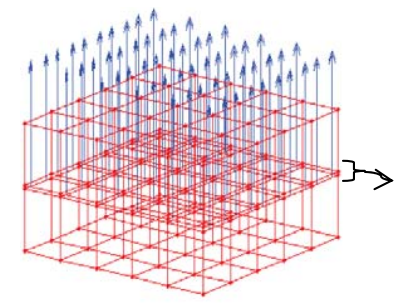

(b) Crack direction

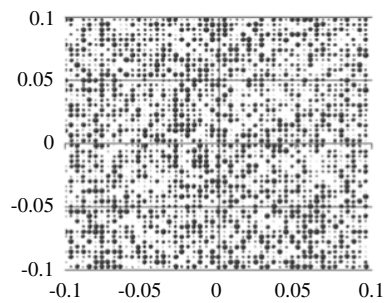

(c) Ideal particle distribution

Figure 9. Interlocking experiment: (a) actual experimental specimen; (b) numerical modeling with initial Mode-I crack, marked by arrows in the middle layer; (c) unstructured random particle distribution over the middle layer of 2,500 elements. (Note: for visualization purpose, 25 and 2,500 elements are shown in (b) and (c), respectively.) 


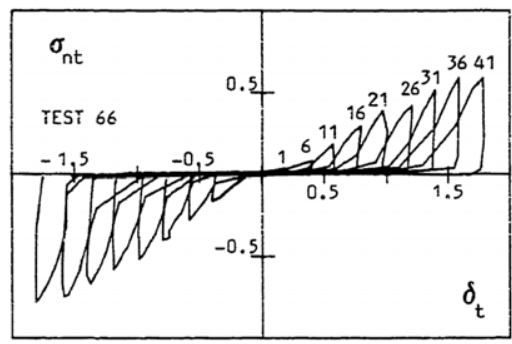

(a) Experiment (from Briseghella and Gori 1984)

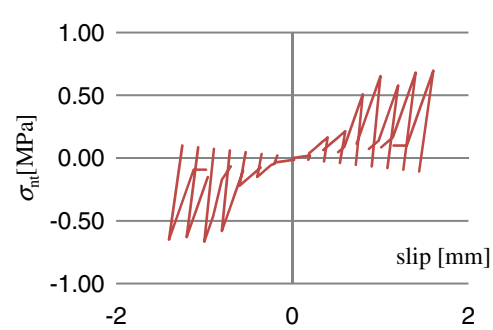

(b) Predicted slip-shear stress

Figure 10. Horizontal slip-resultant force responses (force is represented by corresponding shear stress). The numbers in (a) signify the cycle number recorded during the experiment.

experiments (Eligehausen et al. 1983) in which damage on weak concrete material caused by cyclic loadings is expressed in exponential form.

\section{RANDOM PARTICLE DISTRIBUTION}

When actual RC structures are exposed to seismic loading rather than a discrete crack, multiple crack surfaces are more likely to emerge at random locations, coalesce, and govern the nonlinear shear behavior (Hanson 1996). All of these physical facts led us to the attractive notion of tribology. To describe asperity contact, Jackson and Green (2005) generated random asperity heights from the uncompromised Gaussian distribution for the entire contact surface, and performed integration to draw the resultant global force-slip relationship (cf. similar work by Polycapou and Etsion 1999, Liu et al. 2000). In light of its accuracy, the present platform has adopted the Gaussian distribution as the base probabilistic distribution for generating particle sizes for the 3-D interlocking model.

To clarify the distribution process of the ideal particle, let $D_{h}$ be a homogeneous random field of ideal particle size for the interlocking mechanism in the physical domain Ù. In the present study, U represents the entire domain of structure on which nonlinear degradation is defined. Among various methods (e.g., Li and der Kiureghian 1993), the midpoint method has been adopted to discretize the random field $D_{h}$ into a vector of random variable $D=\left\{D_{1}, D_{2}, \ldots\right\}$ associated with a probability density function $f_{D}$. Following the notion of the midpoint method as done by many (e.g., Carmeliet and de Borst 1995, Gutiérrez and de Borst 1999), a random variable $D_{i}$ defined on a subdomain $\dot{U}_{i} \in \grave{U}$ can be represented by

$$
D_{i}=D_{h}\left(\mathbf{x}_{i}^{c}\right)
$$

where $\mathbf{x}_{i}^{c}$ is a vector representing the center of the subdomain $\dot{U}_{i}$.

Furthermore, we assumed $\dot{U}_{i}$ to be equal to a finite element of the structure under consideration. In this context, each subdomain - that is, a finite element - holds one ideal particle size that is generated from the Gaussian distribution. 


\section{INTERLOCKING DURING CYCLIC MOVEMENT}

Physical interpretation of the interaction between the rigid particle and soft matrix provides clear insight into realistic behavior of cyclic shear resistance. Figure 8 a shows linearly increasing shear stress with the slope induced from the current crack opening, whereas Figure $8 \mathrm{~b}$ shows no active contacting for $0<\gamma<\gamma_{\max }$. Figure 8c describes early development of resistance during reloading. When the current strain lies between the recontacting shear strain $\gamma_{r}$ and the previous maximum strain $\gamma_{\max }$, we reduced shear resistance by $\alpha_{1} G(\tilde{\varepsilon})$, where $\alpha_{1} \in(0,1)$. As reloading further proceeds beyond $\gamma_{\max }$ it is assumed that full contacting has been achieved, and afterward shear stress is regarded to fully obey the interlocking mechanism as depicted in Figure $8 \mathrm{~d}$. Thus at each incremental step, a new tangent shear stiffness $G_{i j}^{c r}$ should be updated from the 3-D interlocking model with current crack strains as

$$
G_{i j}^{c r} \equiv\left\{\begin{array}{cc}
G_{0} & \text { for } \varepsilon_{i}^{c r}, \varepsilon_{j}^{c r} \leq 0 \\
\alpha_{1} G(\tilde{\varepsilon}) & \text { otherwise }
\end{array}\right.
$$

where $\varepsilon_{i}^{c r}=$ normal crack strain in the $i_{t h}$ normal direction on the crack surfaces; $\tilde{\varepsilon}=2 L / D_{\max } \times \max \left(\varepsilon_{i}^{c r}, \varepsilon_{j}^{c r}\right) ; \alpha \in(0,1)$ for $\left|\gamma_{r}\right| \leq|\gamma| \leq\left|\gamma_{\max }\right|$.

\section{VALIDATION AGAINST AN INTERLOCKING EXPERIMENT}

In order to validate the 3-D interlocking model, a well-documented experiment of rough crack by Briseghella and Gori (1984) was selected. In the experimental program, a plain concrete block of dimension $0.2 \times 0.2 \times 0.2 \mathrm{~m}$ is initially separated into two parts, leaving a discrete rough Mode-I crack with width $1 \mathrm{~mm}$. Afterwards, the lower block is horizontally loaded in a reverse cyclic manner by displacement control.

For the numerical simulation, the cracked blocks in Figure 9a were modeled by three layers of finite elements in the vertical direction (each layer consists of 2,500 hexahedral elements). As shall be addressed in the following section, such a large number of elements is not necessarily required. Indeed, if the shearing area is fixed, a small number of elements appears to be enough to obtain the almost identical resultant shear resistance. As shown in Figure 9b, the middle layer was intentionally designed to hold initial Mode-I cracking as well as ensuing interlocking behavior, and the arrows stand for the directions normal to the cracked surface. Initially, ideal particles were generated from the Gaussian distribution and assigned to all elements on the middle layer as shown in Figure 9c.

Figure 10 compares the horizontal slip and resultant force responses from the experiment and prediction. For clarity, the unloading and reloading paths predicted with zero slopes are not shown in Figure 10b, and only ascending and descending paths are shown. As easily seen, the overall nonlinear response of shear force transfer over the rough crack surfaces has been successfully reproduced.

Although not significant, however, some discrepancy is noticeable at large slip range, which appears to be related to the fact that in the simulation, Mode-I crack with an initial opening $(1 \mathrm{~mm})$ was modeled to be smeared within the middle layer of one element height. Unlike the ideal experimental setup with the fixed crack spacing, the cracked solid elements in the middle layer might deform in a way that the Mode-I crack becomes slightly larger than the initial value or than reality. Whereby additional loss of contacting area might happen, thus leading to reduction of peak shear resistance. 


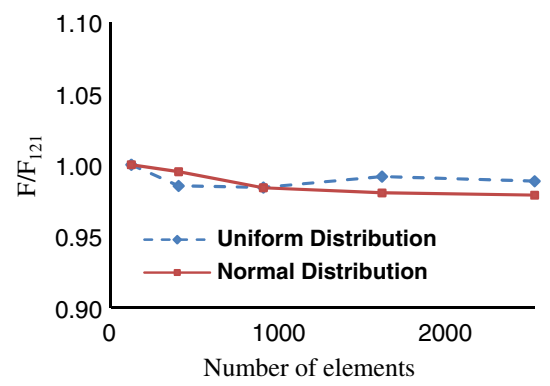

Figure 11. Normalized maximum horizontal forces with varying element numbers for two random particle distributions: normal and uniform distributions.

\section{EFFECT OF NUMBER OF ELEMENTS AND MEAN DIAMETER OF PARTICLES}

With increasing elements through mesh refinement, the diversity of sizes and total number of particles also increases. Figure 11 presents the parametric study on the element size, performed with two representative probabilistic distributions having the same mean diameter $0.019 \mathrm{~m}$ : normal distribution $N\left(0.019,0.00633^{2}\right)$ and uniform distribution $U(0.0001,0.038)$. The aforementioned rough crack experiment is numerically simulated with a varying number of elements, $11^{2} \sim 50^{2}$. The vertical axis of Figure 11 stands for the predicted maximum horizontal forces normalized by that with $11^{2}$ elements. Regardless of the type of probabilistic distribution employed, we can confirm the desired mesh objectivity, provided the total cross-sectional shearing area is fixed.

The mean value used in the probabilistic distribution for random particle size, however, significantly affects the resultant horizontal forces. Figure 12 gives the positive peak forces collected from the numerical simulations of the experiment with initial crack width of $1 \mathrm{~mm}$. As expected, the increased mean size of particle distribution pushes upward the range of forces in a nonlinear manner. It should be noted that with a mean size larger than a certain value - for example, $25 \mathrm{~mm}$ herein - the resulting horizontal forces appear to converge to the specific upper bound, which can be physically conceived by the almost occupied indentations.

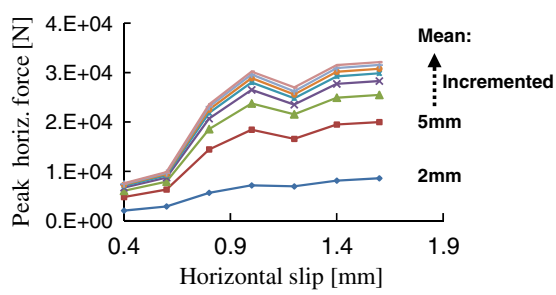

Figure 12. Effect of mean particle size on resultant horizontal force from 3-D interlocking model. 


\section{"SMART" REINFORCING BAR MODEL WITH EVOLVING BUCKLING LENGTH}

Departing from valuable knowledge of the previous studies summarized in the introduction, we aimed to propose a "smart" reinforcing steel model that holds general applicability. In essence, the "smart" reinforcing steel model is a sort of platform, mostly dealing with information about microstates of surrounding materials, topological change (i.e., boundary condition) of the reinforced system, etc., giving rise to a new paradigm of analysis of composite structures. In the near future, further enrichment and sophistication of the platform will be open to researchers in our fields.

To systematically handle the evolution of buckling length of longitudinal steel bars, we define the base unit $B U_{i}$, which consists of the $i_{t h}$ steel bar and a set of its surrounding elements $S_{i}\left(i \in\left[1, n_{s}\right], n_{s}\right.$ the total number of steel bars). In light of topological information, the construction of all units and inter-unit information transfer are automatically carried out in a parallel algorithm (see Cho 2012 for a detailed description and parallelization).

Physically, it is hard to expect the onset of compressive buckling of a longitudinal bar when some portion of the surrounding elements did not crush (or spall). As briefly illustrated in Figure 13, a criterion for evolution of buckling length has been made in such a way that the topological transition can be triggered only when all surrounding elements enter at least partially crushed state. After the transition, we regard bars to lose their surrounding elements and, in turn, they can coalesce with adjacent bars, constructing new base unit pivoting around the lengthened bar.

Topological transition of $B U_{m}$ can be correlated to the dissipated energy of surrounding elements in $S_{m}$ as

$$
\int_{S_{m}} \sigma \mathrm{dV} \geq \mathrm{E}_{\mathrm{th}}
$$

with a specific energy threshold $E_{t h}$. To evaluate the energy state of the material, two internal state variables are introduced: one is defined at the integration point level and the other at the

All surrounding elements of

$\mathcal{S}_{3}, \mathcal{S}_{4}, \mathcal{S}_{5}$ crushed (or spalled)

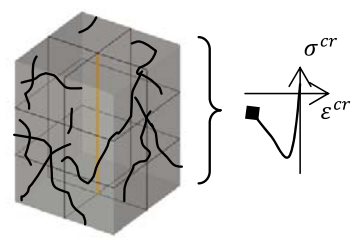

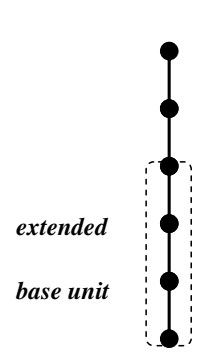

Figure 13. Example of topological transition triggered only when all surrounding elements entered a certain damage level, which in turn lengthened buckling lengths of bars. 
element level. First, let $\alpha_{i}^{(k)}$ be an internal state variable of the $k_{t h}$ crack surface, $k \in\{1,2,3\}$ defined on the $i_{t h}$ integration point, describing uncrushed state by 1 and crushed state by 2

$$
\alpha_{i}^{(k)}=\left\{\begin{array}{cc}
1 & \text { for } \min _{t} \varepsilon_{k}^{c r}>\varepsilon_{t h} \\
2 & \text { otherwise }
\end{array}\right.
$$

with $\varepsilon_{t h}$ strain threshold for entering crush phase, which is assumed in this study as the strain associated with the compressive strength.

Then we can further define an element-level internal variable $\lambda_{j}$, which can signify intact, partially crushed, and fully crushed states of an element by use of simple integer values 0,1 , and 2, respectively. In detail, when all the integration points in an element undergo crushed phase we assign 2 to $\lambda_{j}$. When only some of the integration points in an element enter crushed phase, we regard the element partially crushed denoted by 1 , while intact state of an element is denoted by 0 if none of the integration points experience crushed phase. With the aid of the simple integer-based state variable we have:

$$
\lambda_{j}=\left\{\begin{array}{cc}
0 & \beta_{\mathrm{j}} \leq \mathrm{NINT}^{(j)} \times 3 \\
1 & \mathrm{NINT}^{(j)} \times 3<\beta_{\mathrm{j}} \leq \mathrm{NINT}^{(j)} \times 6 \\
2 & \beta_{\mathrm{j}}>\mathrm{NINT}^{(j)} \times 6
\end{array}\right.
$$

where $\operatorname{NINT}^{(j)}=$ number of integration points of the $j_{t h}$ element $\in S_{m}$ and

$$
\beta_{j}=\sum_{i=1}^{\mathrm{NINT}^{(j)}} \sum_{k=1}^{3} \alpha_{i}^{(k)} .
$$

While these three categories might not be sufficient for fully describing a wide spectrum of damage states, they can effectively detect the most critical states of surrounding concrete materials. Since the internal variable $\lambda_{j}$ is essentially tied to the states definition of advanced material models, further sophistication of the internal variable shall be a natural future research topic.

For the conservative prediction, if all surrounding elements enter the partially crushed phase, the topological transition is assumed to take place, which can be ascertained by

$$
\sum_{j=1}^{n\left(S_{m}\right)} \lambda_{j} \geq \mathrm{n}\left(S_{m}\right)
$$

where $\mathrm{n}\left(S_{m}\right)$ means the number of entities (i.e., surrounding elements) in $S_{m}$. Hence, once the above condition is violated, the topological transition is triggered and the buckling length of the corresponding reinforcing bar can evolve by merging with adjacent bars' buckling lengths, giving new buckling length:

$$
L_{b} \equiv \sum_{L_{0}^{(i)} f o r \forall e_{i}^{1} \in B U_{m}} k L_{0}^{(i)}
$$


where $L_{o}^{(i)}$ is initial buckling length of the $i_{t h}$ steel bar $e_{i}^{1}$, and $k$ is effective length factor set as 0.5 for fixed boundary condition. And, in turn, this evolved buckling length feeds the latest information to the process of new buckling initiation point $\varepsilon^{*}$ as

$$
\frac{\varepsilon^{*}}{\varepsilon_{y}}=55-2.3 \sqrt{\frac{f_{y}}{100}} \frac{L_{b}}{D_{s}} ; \quad \frac{\varepsilon^{*}}{\varepsilon_{y}} \geq 7
$$

where $\varepsilon^{*}$ is the intermediate strain where buckling initiates, following the key notion of Dhakal and Maekawa (2002a); $\varepsilon_{y}$ and $f_{y}$ are the strain and stress $[\mathrm{MPa}]$ at yielding; $L_{b}$ is the buckling length updated; and $D_{s}$ is the diameter of the bar cross section. The suggested bar model explained so far is schematically illustrated in Figure 14.

Figure 15 compares the analytical prediction with the well-known experiment of single bar (Monti and Nuti 1992). The analytical response exhibits overall success in capturing compressive buckling phenomena over a wide range of $L / D$ ratio. It is noteworthy, however, that the lack of some advanced aspects - such as flat plateau regime after initial yielding, smooth transition to buckling, and so on - appears to exert influence on the noticeable discrepancy (e.g., the case of $L / D=11$ in Figure 15). Such sophisticated features are readily available in literature (e.g., Kunnath et al. 2009), and their inclusion shall be a natural future extension.

To further understand the evolving buckling length, a simple reinforced concrete system is simulated by cyclic vertical loading as illustrated in Figure 16a. Relatively weak compressive strength is assigned to one or two bottom layers (Figure 16b) in hopes of confirming the

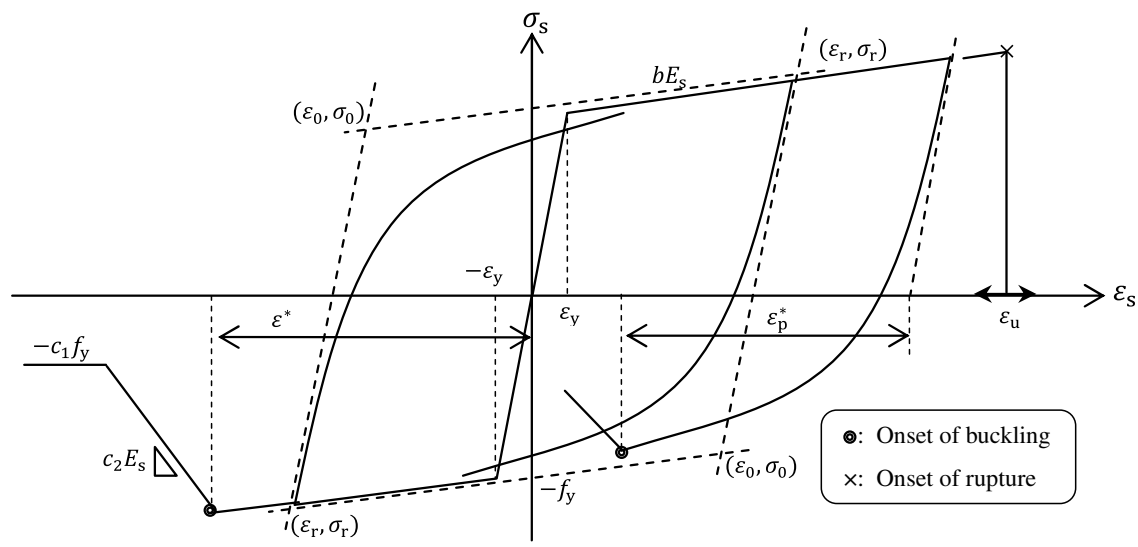

Figure 14. Generalized Menegotto-Pinto bar model capturing compressive buckling. The parameter $b$ is for stiffness reduction after exceeding yielding point $\left(\varepsilon_{y}, f_{y}\right) .\left(\varepsilon_{r}, \sigma_{r}\right)$ is the point where load reversal happens while $\left(\varepsilon_{0}, \sigma_{0}\right)$ means the intersection point between lines of initial and reduced stiffness. The ultimate strain $\varepsilon_{u}$ signifies tensile rupture. Following Rodriguez et al. (1999), $\varepsilon_{p}^{*}$ is introduced for capturing early buckling at positive strain after large tensile loading. The parameters $c_{1}$ and $c_{2}$ are recommended to be 0.2 and 0.02 by Dhakal and Maekawa (2002a). 

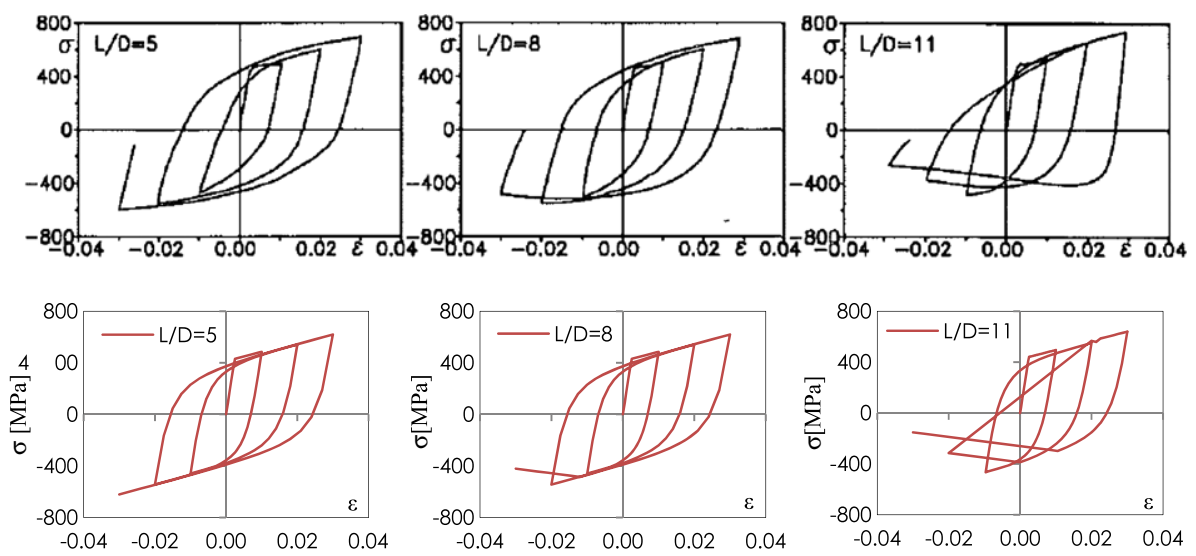

Figure 15. Comparison between experimental diagram (top row, from Monti and Nuti 1992) and analytical results from the proposed bar model (bottom row), confirming the overall success in capturing compressive buckling phenomena over various $L / D$ ratios.

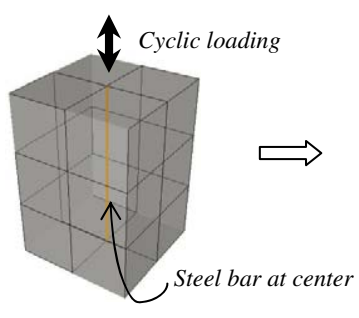

(a)

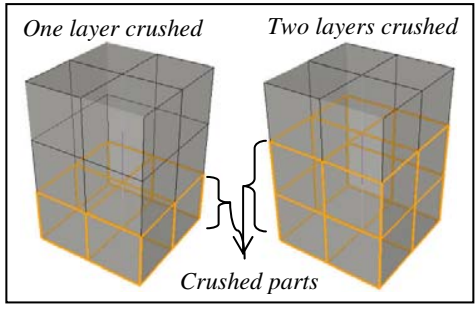

(b)

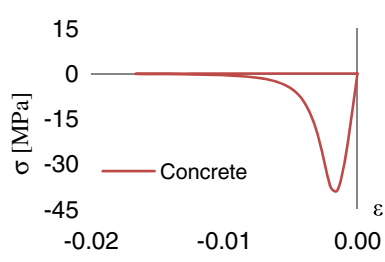

(c)

Figure 16. Illustration of sample study on different buckling modes, for which weak material property of concrete has been assigned to different parts of the structure: (a) cyclically loaded test setup of three layers of concrete with vertical steel bar at center (initial $L / D$ of each bar element at one layer is 5); (b) one or two layers is made to quickly enter into crushed phase; (c) example result of concrete stress-strain history showing the excessively crushed state.

evolving buckling length in accordance with concrete crushing (Figure 16c), and thus clear contrast in buckling modes.

Figure 17a shows clear contrast in bar buckling modes. When the two concrete layers enter into the crushed phase, the ratio $L / D$ also automatically evolves to 10 , twice the initial value of 5 . Thus, the loss of two concrete layers causes far earlier bar buckling than the case with one layer being crushed. Another simulation result presented in Figure 17b assures the successful prediction of the early initiation of buckling after transversal from excessive tensile loading, as suggested by Rodriguez et al. (1999). The significant role of evolving buckling length is obvious in the early emergence of buckling. 


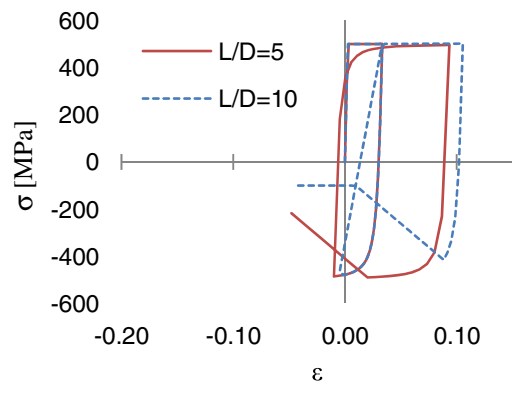

(a)

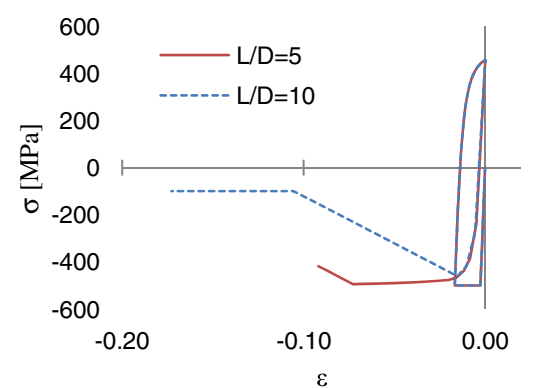

(b)

Figure 17. Clear contrast in compressive buckling modes of bar induced by automatically evolving buckling length: (a) when two layers of concrete are crushed, the ratio $L / D$ evolved to 10 , twice the initial value of 5 , thus causing the compressive buckling of bar to happen earlier than the case with one layer being crushed; (b) early buckling after reversal from excessive tensile loading is largely affected by the evolved buckling length.

\section{APPLICATIONS TO LARGE-SCALE STRUCTURES}

\section{H-SHAPED WALL SYSTEM UNDER CYCLIC LOADING}

An experiment of real scale RC structure exhibiting sufficient complexity of both geometry and nonlinear behavior had been selected: Model DP1, the three-dimensional H-shaped wall system under cyclic loading conducted by Palermo and Vecchio (2002).

In both web and flange, the concrete strength is $21.7 \mathrm{MPa}$ and the strain at the peak is 0.00204. All bars are D6 type with 7-mm diameter, and the yielding stress and the corresponding strain are $605 \mathrm{MPa}$ and 0.00318 , respectively. All the reinforcing bars are explicitly modeled by space truss elements with "perfectly bonded" assumption. The finite element model consists of 7,784 solid elements and 11,212 nodes. Figure 18 shows the dimensional details of DP1. It is noteworthy that this particular experimental program contains no horizontal stirrups/hoops.

To successfully utilize the 3-D interlocking model for this particular experiment, ideal particles were generated from the Gaussian distribution, for which the mean value was $19 \mathrm{~mm}$ (ranging from $0.1 \mathrm{~mm}$ to $38 \mathrm{~mm}$ ). Then they were randomly distributed over the entire domain as depicted in the inset of Figure 18.

According to Figure 19a, without the 3-D interlocking model, the simulation predicts overstiff responses as expected, which indeed confirms the pathological nature of the fixed-type smeared crack model at real scale structure level. Contrariwise, Figure 19b asserts that the positive contribution of the 3-D interlocking mechanism to the smeared crack model is remarkably significant. The hardening behavior beyond peak point (i.e., displacement loading greater than $\pm 8 \mathrm{~mm}$ ) is markedly alleviated by the interlocking mechanism, showing successful agreement with experimental response.

Remarkably, material heterogeneity of domain introduced by the 3-D interlocking model appears to be correlated with the emergence of localized damage. In the absence of the 3-D interlocking model, damage induced by the smeared cracking appears to be so smoothly 


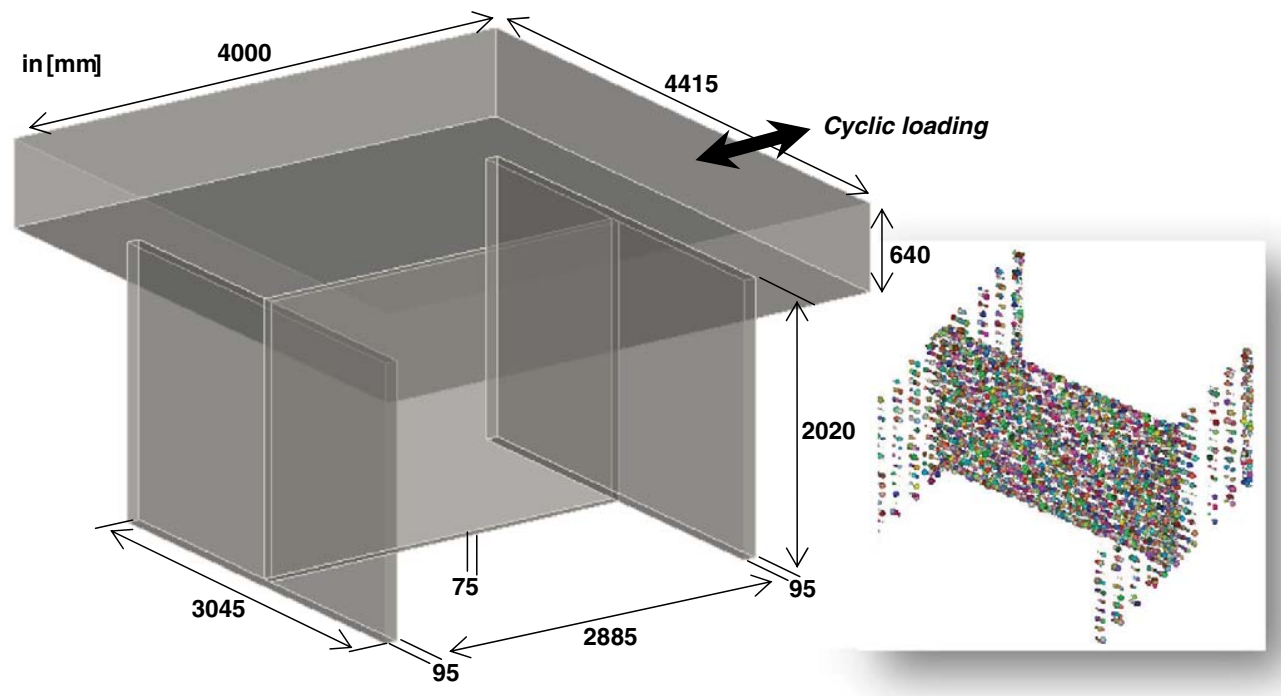

Figure 18. H-shaped wall system details and random particle distribution for modeling irregular asperity configuration of crack surfaces (inset).

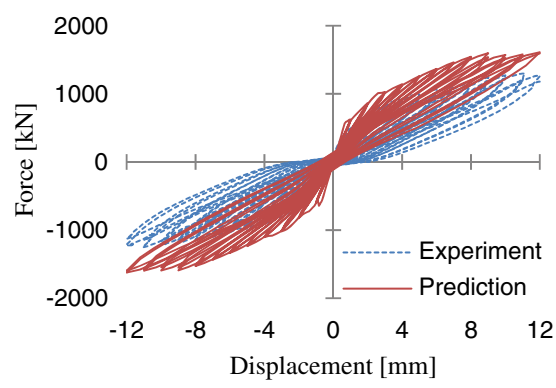

(a) Without interlocking model

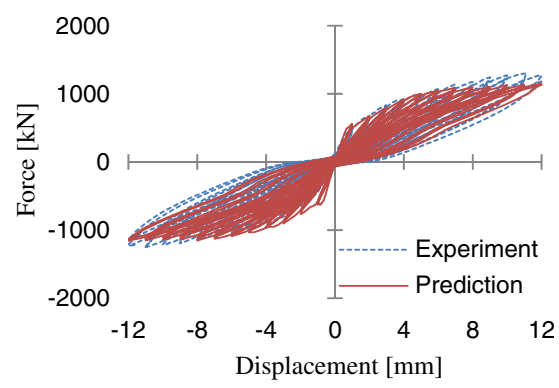

(b) With interlocking model

Figure 19. Effect of 3-D interlocking model on the global force-displacement responses (simulated up to $12 \mathrm{~mm}$ near the peak load-carrying capacity).

scattered over the wall system that it rarely leads to any noticeable formation of the localized damage (Figure 20a). By introducing 3-D interlocking, however, the simulation allows the development of noticeable localized damage, notably in the form of out-of-plane bulging with vertical directivity on the web part as marked by the dashed line in Figure 20b. Indeed, the localized damage with vertical directivity is one of the unique features of this particular experimental program (probably due to relatively thin web thickness, coarse reinforcing bars on the web, excessively wide flanges, etc.), while a typical shear wall system is normally failed with diagonal directivity. The random particles over the "entire" domain are believed to be essential to capture such a unique localized damage (cf. comparable discoveries in the heterogeneous granular medium by Shahinpoor 1980 and Andrade et al. 2007). 


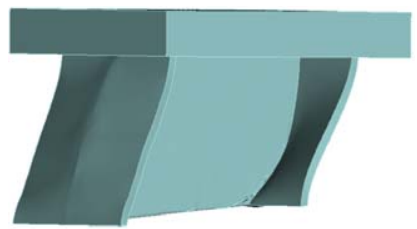

(a) Without interlocking model

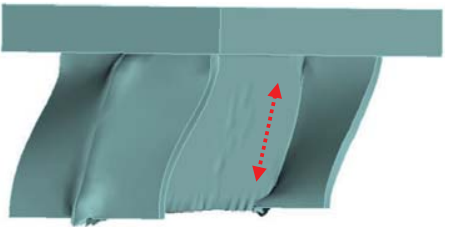

(b) With interlocking model

Figure 20. Deformed shape (amplified) with/without 3-D interlocking model. (Note: By introducing the 3-D interlocking mechanism, the localized damage with vertical directivity marked in dashed arrow can be captured.)

\section{FOUR-STORY T-SHAPED WALL SYSTEM UNDER CYCLIC LOADING}

The next application used for validation is more enriched by asymmetric geometry and sophistication of reinforcing steels - the four-story T-shaped wall system (Thomsen IV and Wallace 1995, 2004). The wall system was loaded by cyclic displacement up to its ultimate damage states - namely, fully developed diagonal cracks on web, and crushing and spalling of concrete accompanied by compressive buckling of longitudinal reinforcing bars.

The experimental program, denoted by TW2 hereafter, is approximately one-quarter scale of part of the original prototype building, similar to a typical multistory office structure in a high seismicity zone such as Los Angeles. Along with random particle distribution over the entire domain, the detailed geometric information is given in Figure 21. TW2 is $3.66 \mathrm{~m}$ tall and $102 \mathrm{~mm}$ thick, and the length of both web and flange is $1.22 \mathrm{~m}$. Stiff floor is placed at 914-mm intervals.

The strength of concrete and corresponding strain are $41.7 \mathrm{MPa}$ and 0.002 , respectively. Tensile strength is $5.63 \mathrm{MPa}$. The yield strength and corresponding strain are commonly $414 \mathrm{MPa}$ and 0.00207 for longitudinal steels (Grade 60 No. 3 and No. 2) and hoops and ties $(\mathrm{D}=4.75 \mathrm{~mm})$, and more detailed properties of reinforcing steels can be found elsewhere (Thomsen IV and Wallace 1995, 2004). In total, 67,410 linear hexahedral elements for concrete and 20,646 space truss elements for all reinforcing bars are generated (i.e., 84,211 nodes). It should be stressed that no artificial separation between cover and core concrete, which is normal in popular fiber-section models, has been included.

Figure 22 compares the resultant force-displacement plots from predictions with and without 3-D interlocking model; the upper and lower limits of the recorded force range from the experiment are given by the dashed lines for comparison. Overall, the 3-D interlocking model appears to successfully coordinate with the fixed-type smeared crack model in the simulation, enforcing the range of load-carrying capacity to be accurately bounded (Figure 22a). Contrariwise, without 3-D interlocking mode, the overstiff nature of the smeared crack model remains clearly visible (Figure 22b). Such overestimated response seems more apparent in the positive displacement range (i.e., flange under compression) than in the negative range (i.e., flange under tension). In the case of the flange under compression, with asymmetric placement of the wide flange, a large portion of the bottom web undergoes tension and shear-governed deformation. This can be restated in more practical terms such that the so-called neutral axis of the bottom section is located near the wide 


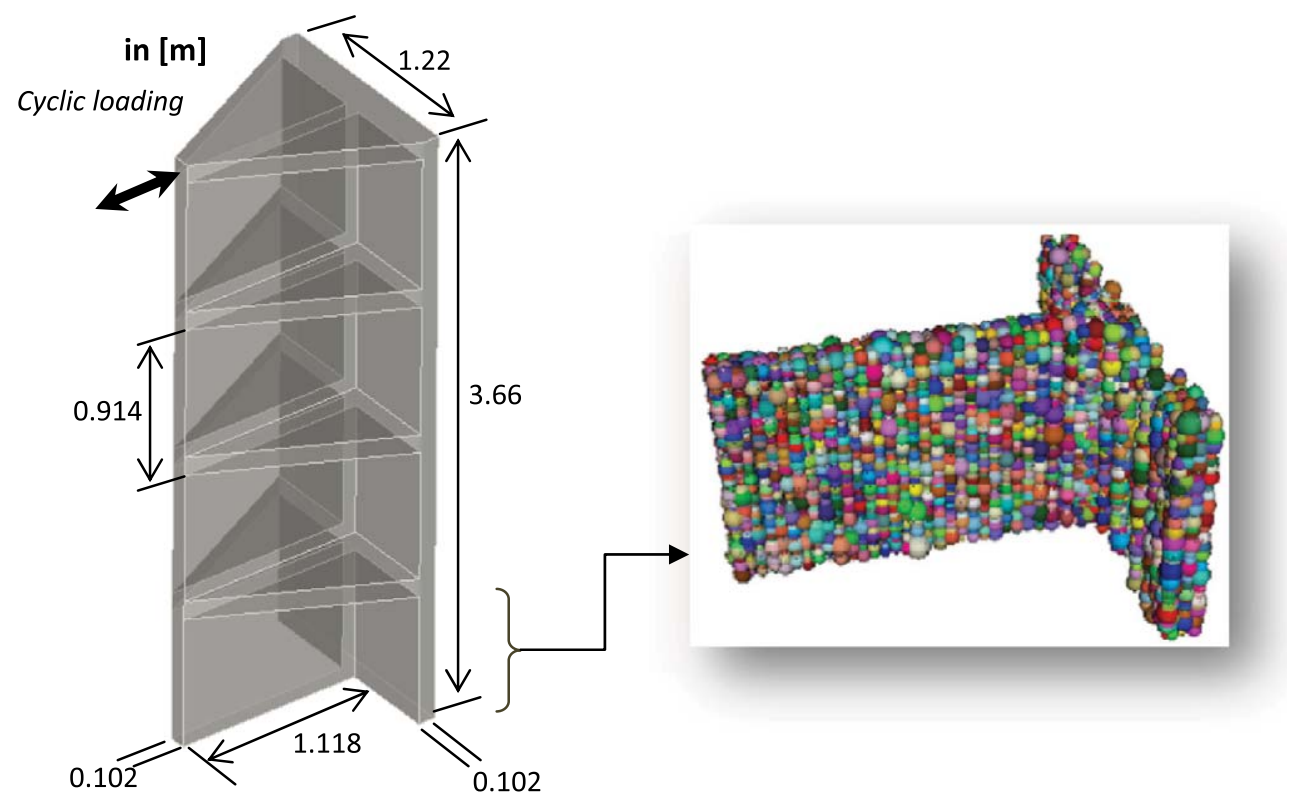

Figure 21. Overall geometry of TW2 and (inset) random particle distribution over entire domain for modeling irregular asperity configuration of crack surfaces.

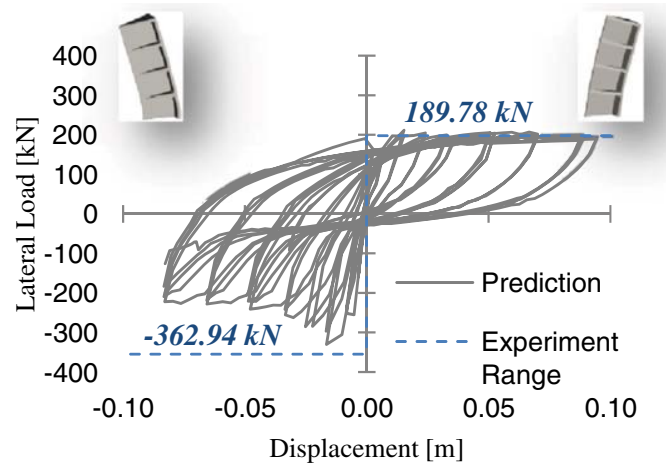

(a) With interlocking model

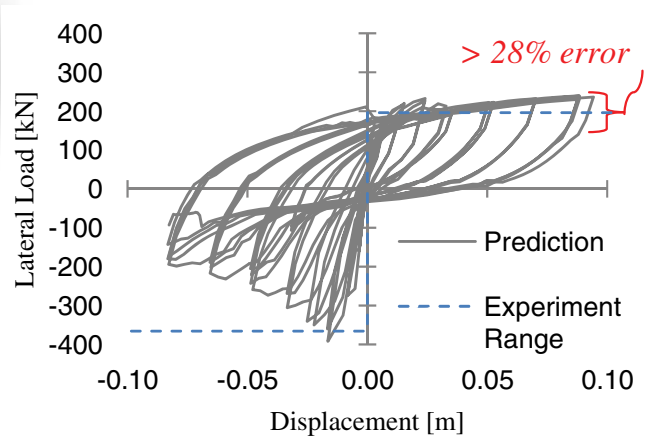

(b) Without interlocking model

Figure 22. Effect of 3-D interlocking model on global force-displacement response (numbers next to dashed line show the maximum lateral load range from the experiment): (a) with the interlocking model, well-bounded lateral load response has been generated, whereas (b) without interlocking model, error is escalating (exceeding even $28 \%$ ) and unboundedly increasing. Both cases commonly underestimate (in negative range) load-carrying capacity due mainly to the exclusion of confinement effect consideration. 
flange, causing web under tension and flange under compression (Thomsen IV and Wallace 2004). Therefore, the interlocking mechanism, which always manifests itself in the presence of actively opened cracks, significantly influences the global resistance when the flange is under compression (i.e., positive displacement regime in Figure 22). On the other hand, when the flange is under tension (negative displacement range), both predictions with and without 3-D interlocking model appear to commonly underestimate the load-carrying capacity of the system compared to the experimental record. This common underestimation is most likely tied to lack of consideration of confinement effect in both simulations. In particular, despite the full realization of reinforcing system, the compressive stress is still updated from the microscopic stress function, and relatively large compressive strain can develop in the web due to the wide width of the flange, together producing weak compressive stress from the postpeak regime. Thus it would be a natural extension in the future research to incorporate appropriate physical mechanisms capable of describing lateral confinement effect into the present framework.

As shown in Figure 23, it is fascinating to capture the irrecoverable damage, notably in a localized manner. Emphasized by dashed arrows in the figure, the damage is concentrated at the web part with apparent diagonal directivity. Intrinsic randomness of particles in 3-D interlocking model is believed to have a strong influence on such a localized damage of the web. Furthermore, compressive crushing at the web toe is also well predicted and shown in a somewhat exaggerated manner.

It is apparent that longitudinal steel placed at the outermost position of web toe is experiencing progressive buckling according to the excessively large compressive strain as shown
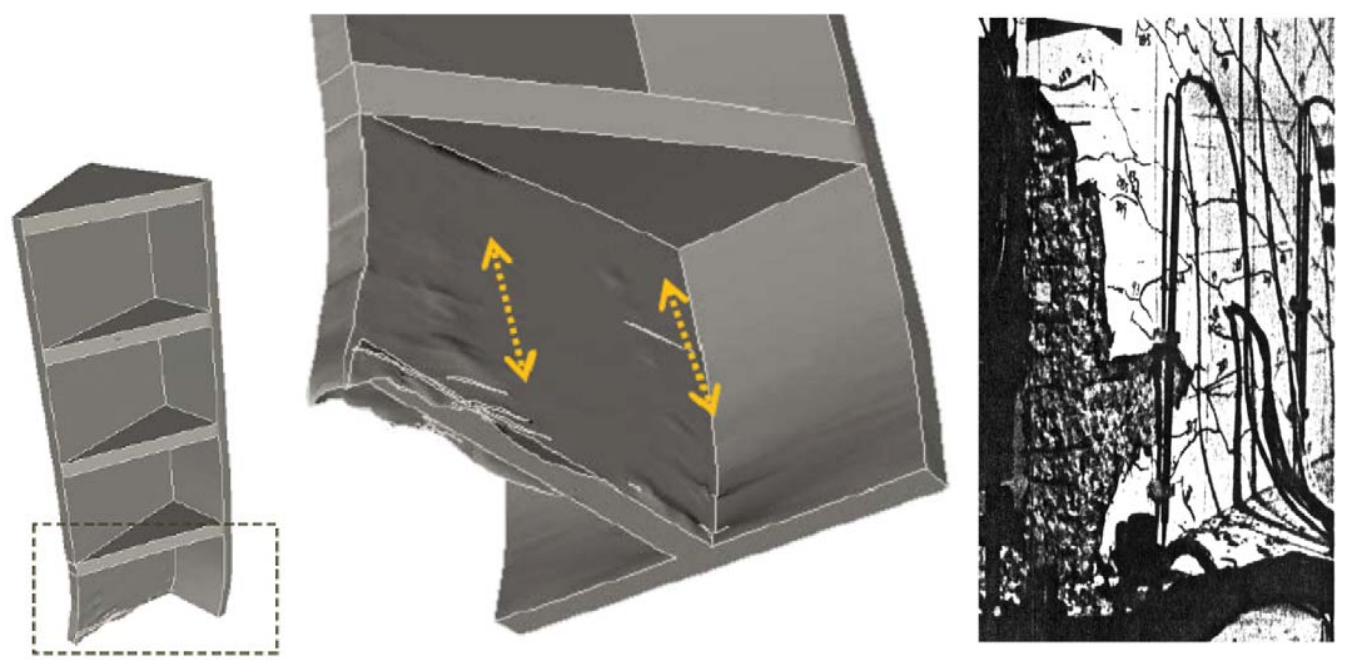

Figure 23. Deformed shape (amplified) at the last step of the simulation, revealing concentrated severe damage at web boundary part and the localized damage with diagonal directivity (marked by dashed arrows) by virtue of the 3-D interlocking model. In the right photo, actual failure state with severe damage at web and diagonal cracks is provided for comparison (photo from Thomsen IV and Wallace 1995). 


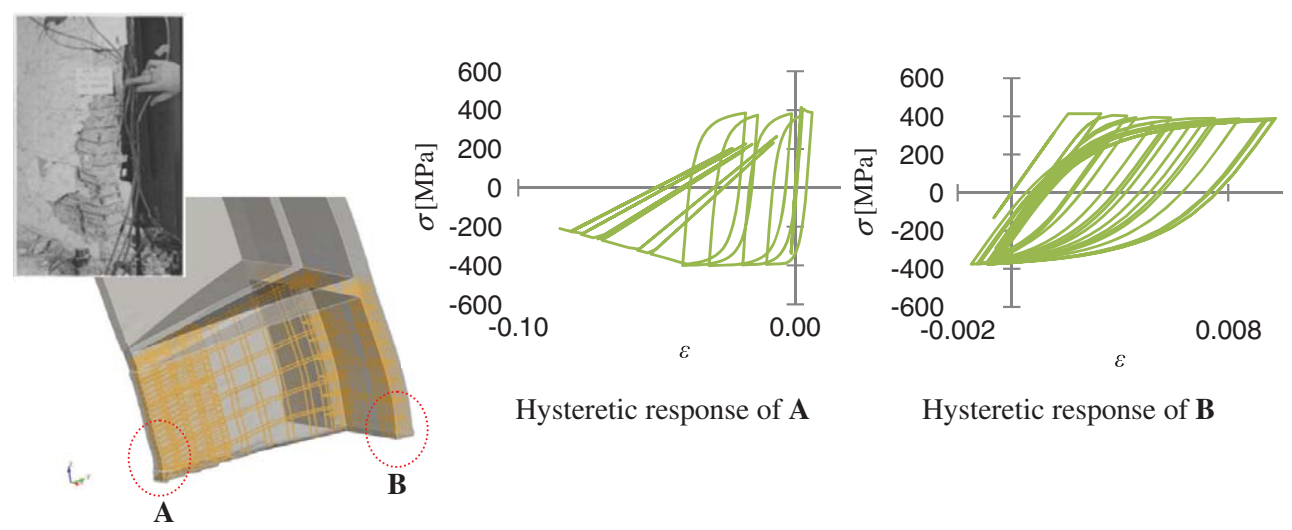

Figure 24. Apparent contrast in the predicted hysteretic responses of longitudinal steel bars depending on their locations; that is, we confirm buckling of bar located at the outermost positions of web toe (denoted A) with excessive compressive strain, whereas no buckling at the bar of flange (denoted B). Inset at left top is the actual photo with spalling and crushing over a large number of tie spacings, which bears out the progressive buckling of longitudinal bars (inset from Thomsen IV and Wallace 2004).

in Figure 24. Contrarily, from the bars at the flange, typical stress-strain response with smooth transition after yielding is identified, with relatively small strain range $(<0.01)$. The predicted compressive buckling length is well consistent with the realistic failure mode (inset of Figure 24) of the region near web toe experiencing severe crushing and spalling, notably over a large number of tie spacings.

\section{CONCLUSIONS}

We presented a novel parallel simulation platform to settle the unresolved questions as to nonlinear shear, localized damage and progressive buckling of reinforcing bars. Inspired by tribology, we adopted the fabric of rigid particle-soft matrix, of which interaction generates nonlinear shear stress on crack surfaces under cyclic loading. For multiple rough crack surfaces, we distribute random particles from the Gaussian distribution over the "entire" domain. Validation of the 3-D interlocking model against an experiment reveals remarkable accuracy and possibility of the model.

Departing from well-established previous researches, we presented a platform for a "smart" steel bar model. With the aid of an automated and parallelized platform, comprehensive consideration of nonlinear degradation of the reinforced system of bar and surrounding materials offered a smart platform to capture the progressive buckling phenomena.

Simulation of real scale structures with the aid of the parallel platform is itself opening new possibilities of "top-down" validation, offering a clear causal pathway between underlying physical mechanisms and the particular global response. From the large-scale applications, the well-known pathological nature of the fixed-type smeared crack model has been successfully resolved by the inclusion of a 3-D interlocking mechanism. Notably, the 
randomness of salient parameters over the entire domain appears to play an essential role to cause localized damage.

\section{ACKNOWLEDGMENTS}

Regarding experimental data for validation, the warm hospitality of Professor Daniel Palermo, Professor Frank J. Vecchio, and Professor John W. Wallace is appreciated. All numerical simulations related to the present work were run on GARUDA, a high-performance computing cluster hosted within the civil engineering department at Caltech. The purchase and installation of GARUDA was in large part possible thanks to the Ruth Haskell Research Fund, the Tomiyasu Discovery Fund, and Dell Inc. Professor S. Krishnan's warm support with the cluster is deeply appreciated. Special thanks are due to Professor J. F. Hall for his consistent support and productive discussion.

\section{REFERENCES}

Adebar, P., and Ibrahim, A. M. M., 2002. Simple nonlinear flexural stiffness model for concrete structural walls, Earthquake Spectra 18, 407-426.

Andrade, J. E., Baker, J. W., and Ellison, K. C., 2008. Random porosity fields and their influence on the stability of granular media, Int. J. Numer. Methods Eng. in Geomechanics 32, 1147-1172.

Bae, S., Mieses, A. M., and Bayrak, O., 2005. Inelastic buckling of reinforcing bars, J. Struct. Eng. 131, 314-321.

Bažant, Z. P., and Oh, B. H., 1983. Crack band theory for fracture of concrete, Matériaux et Constructions 16, 155-177.

Briseghella, L., and Gori, R., 1984. Aggregate interlock cyclic response of R.C. critical section, Proc., 8th World Conf. on Earthquake Engineering, San Francisco, Prentice-Hall Inc., Englewood Cliffs, N.J.

Carmeliet, J., and de Borst, R., 1995. Stochastic approaches for damage evolution in standard and non-standard continua, Int. J. Solids Structures 32, 1149-1160.

Cho, I., 2012. Virtual Earthquake Engineering Laboratory with Physics-Based Degrading Materials on Parallel Computers, Ph.D. Thesis, California Institute of Technology, Pasadena, CA.

Cho, I. H., and Hall, J. F., 2012. A parallelized implicit nonlinear FEA program for real scale RC structures under cyclic loading, Journal of Computing in Civil Engineering 26, 356-365.

Cope, R. J., Rao, P. V., Clark, L. A., and Norris, P., 1980. Modeling of reinforced concrete behavior for finite element analysis of bridge slabs, Numerical Methods for Nonlinear Problems I (Taylor, ed.), Pineridge Press, Swansea, 457-470.

de Borst, R., 2001. Some recent issues in computational failure mechanics, Int. J. Numer. Methods Eng. 52, 63-95.

de Borst, R., and Nauta, P., 1985. Non-orthogonal cracks in a smeared finite element model, Engineering Computations 2, 35-46.

Dhakal, R., and Maekawa, K., 2002a. Modeling for postyielding buckling of reinforcement., J. Struct. Eng. 128, 1139-1147.

Dhakal, R., and Maekawa, K., 2002b. Reinforcement stability and fracture of cover concrete in reinforced concrete members, J. Struct. Eng. 128, 1253-1262.

Elkadi, A. S. K., 2005. Fracture Scaling of Concrete under Multiaxial Compression. Ph.D. thesis, Delft University of Technology, the Netherlands. 
Eligehausen, R., Popov, E. P., and Bertero, V. V., 1983. Local Bond Stress-Slip Relationships of Deformed Bars under Generalized Excitations, EERC Report, UCB/ EERC-83/23.

Greenwood, J. A., and Williamson, J. B. P., 1966. Contact of nominally flat surfaces, Proc. R. Soc. A 295, 300-319.

Gutiérrez, M. A., and de Borst, R., 1999. Numerical analysis of localization using a viscoplastic regularization: Influence of stochastic material defects, Int. J. Numer. Methods Eng. 44, 1823-1841.

Hanson, R. D., 1996. Evaluation of reinforced concrete members damaged by earthquakes, Earthquake Spectra 12, 457-478.

Hicher, P. Y., 2008. Constitutive Modeling of Soils and Rocks (Hicher, Pierre-Yves, and Shao, Jian-Fu, eds.), ISTE Ltd. and John Wiley \& Sons, Inc., 143-185.

Hsu, T. T. C., and Mansour, M. Y., 2005. Stiffness, ductility, and energy dissipation of rc elements under cyclic shear, Earthquake Spectra 21, 1093-1112.

Jackson, R. L., and Green, I., 2006. A statistical model of elasto-plastic asperity contact between rough surfaces, Tribology International 39, 906-914.

Jirásek, M., 1998. Embedded crack models for concrete fracture. Computational Modelling of Concrete Structures, Balkema, Rotterdam.

Jirásek, M., and Zimmermann, T., 1998. Rotating crack model with transition to scalar damage, Journal of Engineering Mechanics 124, 277-284.

Kunnath, S. K., Heo, Y. A., and Mohle, J. F., 2009. Nonlinear uniaxial material model for reinforcing steel bars, J. Struct. Eng. 135, 335-343.

Li, C. C., and der Kiureghian, A., 1993. Optimal discretization of random fields, J. Eng. Mech. 119, 1136-1154.

Liu, Z., Neville, A., and Reuben, R. L., 2000. Analytical solution for elastic and elastic-plastic contact models, Trib. Trans. 43, 627-634.

Mansour, M. Y., and Hsu, T. T. C., 2005. Behavior of reinforced concrete elements under cyclic shear: Part II - Theoretical model, J. Struct. Eng. 131, 54-65.

Monti, G., and Nuti, C., 1992. Nonlinear cyclic behavior of reinforcing bars including buckling, J. Struct. Eng. 118, 3268-3284.

Orakcal, K., and Wallace, J. W., 2006. Flexural modeling of reinforced concrete walls - experimental verification. ACI Structural Journal 103, 196-206.

Ortiz, M., 1987. An analytic study of the localized failure modes of concrete, Mechanics of Materials 6, 159-174.

Palermo, D., and Vecchio, F. J., 2002. Behavior of three-dimensional reinforced concrete shear walls, ACI Structural Journal 99, 81-89.

Pantazopoulou, S. J., 1998. Detailing for reinforcement stability in RC members, J. Struct. Eng. 124, 623-632.

Peerlings, R. H. J., de Borst, R., Brekelmans, W. A. M., and de Vree, H. P. J., 1996. Gradient-enhanced damage for quasi-brittle materials, Int. J. Numer. Methods Eng. 39, 3391-3403.

Polycarpou, A. A., and Etsion, I., 1999. Analytical approximations in modeling contacting rough surfaces, ASME J. Tribol. 121, 234-239.

Rashid, Y. R., 1968. Ultimate strength analysis of prestressed concrete pressure vessels, Nuclear Engineering and Design 7, 334-344. 
Regan, P., 1971. Shear in Reinforced Concrete: An Experimental Study, Report to the Construction Industry Research and Information Association, Imperial College, Department of Civil Engineering, London, England, 93-110.

Reinhardt, H. W., 1984. Fracture mechanics of an elastic softening material like concrete, Heron 29.

Rodriguez, M. E., Botero, J. C., and Villa, J., 1999. Cyclic stress-strain behavior of reinforcing steel including effect of buckling, J. Struct. Eng. 125, 605-612.

Rots, J. G., 1988. Computational Modeling of Concrete Fracture, Ph.D. thesis, Delft University of Technology, the Netherlands.

Selby, R. G., and Vecchio, F. J., 1993. Three-dimensional Constitutive Relations for Reinforced Concrete, Tech. Rep. 93-02, University of Toronto, Department of Civil Engineering, Toronto, Canada.

Selby, R. G., and Vecchio, F. J., 1997. A constitutive model for analysis of reinforced concrete solidm Can. J. Civ. Eng. 24, 460-470.

Shahinpoor, M., 1980. Statistical mechanical considerations on the random packing of granular materials, Powder Technology 25, 163-176.

Simo, J. C., and Ju, J. W., 1987a. Strain- and stress-based continuum damage models - I. Formulation, Int. J. Solids Structures 23, 821-840.

Simo, J. C., and Ju, J. W., 1987b. Strain- and stress-based continuum damage models - II. Computational aspects, Int. J. Solids Structures 23, 841-869.

Steglich, D., Pirondi, A., Bonora, N., and Brocks, W., 2005. Micromechanical modelling of cyclic plasticity incorporating damage, Int. J. Solids Structures 42, 337-351.

Taucer, F., Spacone, E., and Filippou, F. C., 1991. A Fiber Beam-Column Element for Seismic Response Analysis of Reinforced Concrete Structures, EERC Report, UCB/EERC-91/17.

Thomsen IV, J. H., and Wallace, J. W., 1995. Displacement-Based Design of Reinforced Concrete Structural Walls: Experimental Studies of Walls with Rectangular and T-Shaped Cross Sections, Rep. No. CU/CEE-95/06, Department of Civil and Environmental Engineering, Clarkson University, Potsdam, New York.

Thomsen IV, J. H., and Wallace, J. W., 2004. Displacement-based design of slender reinforced concrete structural walls-experimental verification, J. Struct. Eng. 130, 618-630.

Thorenfeldt, E., Tomaszewicz, A., and Jensen, J. J., 1987. Mechanical properties of high-strength concrete and applications in design, Proc. Symp. Utilization of High-Strength Concrete, Stavanger, Norway.

Vecchio, F. J., 1999. Towards cyclic load modeling of reinforced concrete. ACI Structural Journal 96, 193-202.

Vecchio, F. J., and Collins, M. P., 1986. The modified compression field theory for reinforced concrete elements subjected to shear, ACI Journal Proceedings 83, 219-231.

Weihe, S., Kröplin, B., and de Borst, R., 1998. Classification of smeared crack models based on material and structural properties, Int. J. Solids Structures 35, 1289-1308.

Walraven, J. C., 1994. Rough cracks subjected to earthquake loading, J. Struct. Eng. 120, $1510-1524$.

Yazgan, U., and Dazio, A., 2011. Simulating maximum and residual displacements of rc structures: I. Accuracy, Earthquake Spectra 27, 1187-1202.

(Received 4 October 2011; accepted 19 February 2012) 